\title{
Detección ecográfica y pronóstico de la ventriculomegalia fetal
}

\author{
Mercedes Hernández S. ${ }^{1}$, Olivia Orribo M. ${ }^{1}$, Ingrid Martínez W. ${ }^{2}$, Ana I. Padilla P.2, \\ Margarita Álvarez de la Rosa $R^{2}$, Juan M. Troyano $L .3$
}

1 Médico Residente Ginecología y Obstetricia, ${ }^{2}$ Médico Adjunto Ginecología y Obstetricia, ${ }^{3}$ Unidad de Ecografía. Servicio de Obstetricia y Ginecología, Hospital Universitario de Canarias, Universidad de La Laguna, Tenerife, España.

\section{RESUMEN}

Antecedentes: La ventriculomegalia fetal es un signo ecográfico muy sensible para las alteraciones del sistema nervioso central fetal. La morbilidad asociada a la misma depende principalmente de la etiología y de la presencia de otras malformaciones asociadas. Si es aislada y no progresiva se puede preveer un resultado posnatal favorable hasta en el $70 \%$ de los casos aunque hay una gran disparidad de cifras entre distintos centros y autores. Objetivo: Establecer la relación entre ventriculomegalia y pronóstico perinatal. Método: Se han revisado 60 casos diagnosticados mediante ecografía prenatal en el Hospital Universitario de Canarias siguiendo los criterios ecográficos de ventriculomegalia (astas posteriores mayores de $10 \mathrm{~mm}$ ). Se realizaron cariotipos y serología materna y en líquido amniótico para completar el estudio. Resultados: Hubo un total de 23 interrupciones voluntarias de la gestación y 28 recién nacidos sin secuelas $(47 \%)$. Un $70 \%$ de las ventriculomegalias aisladas correspondieron a recién nacidos sin secuelas. El 10\% de las ventriculomegalias aparecieron en fetos con aneuploidías. Conclusión: El pronóstico fue favorable en los casos de ventriculomegalia no progresiva y aislada. La medida de la ventriculomegalia no predice el resultado. La variabilidad de resultados en la literatura obliga a cada centro a disponer de sus protocolos y datos para aconsejar a la pareja que ha de tomar una decisión sobre el futuro de su embarazo.

\section{PALABRAS CLAVE: Diagnóstico prenatal, ventriculomegalia, ecografía, aneuploidías}

\section{SUMMARY}

Background: Fetal ventriculomegaly is the most sensitive sonographic sign for central nervous system anomalies. Neonatal morbidity and mortality depends on the etiology of the ventriculomegaly and the presence of other malformations. Isolated non-progressive ventriculomegaly is associated with $70 \%$ favorable results, with a great disparity among studies. Aims: To establish the relationships between ventriculomegaly and perinatal outcome. Methods: The authors reviewed 60 cases of prenatally diagnosed fetal ventriculomegaly in the Canary Islands University Hospital. An ultrasonographic measurement of the lateral ventricles of 10 $\mathrm{mm}$ or more was considered as ventriculomegaly. Fetal and maternal serology was performed as well as fetal karyotyping. Results: 23 patients decided to terminate the pregnancy due to unfavorable prognosis. In 28 cases outcome was favorable. $70 \%$ of isolated ventriculomegaly corresponded to healthy newborns. $10 \%$ of the fetuses had aneuploidies. Conclusion: Prognosis is favorable if ventriculomegaly is isolated and nonprogressive. Measurement of the lateral ventricle does not predict outcome. Disparity of results in literature makes it necessary for each Hospital to rely on their own data and guidelines to assess parents on the fate of the pregnancy.

KEY WORDS: Prenatal diagnosis, ventriculomegaly, ultrasound, aneuploidy 


\section{INTRODUCCIÓN}

La ventriculomegalia es un signo ecográfico que puede asociar un número elevado de entidades patológicas con pronóstico propio; es el marcador más sensible de la patología del sistema nervioso central fetal. Determinar la causa de la ventriculomegalia conlleva una tarea compleja $(1,2)$. La morbilidad asociada, depende principalmente de la etiología y presencia de otras malformaciones asociadas. Existe controversia entre la diferencia de este término con el de hidrocefalia, que para algunos autores hace referencia a la etiología obstructiva (3). Siguiendo protocolos internacionales, se define ventriculomegalia como un diámetro de las astas posterior de los ventrículos laterales (atrio) entre $10-15 \mathrm{~mm}$ en el período comprendido entre las 15-40 semanas $(2,4)$. La medida se tomará siguiendo el eje del ventrículo perpendicular a sus paredes, medida de interior a interior, según recomendaciones la International Society of Ultrasound in Obstetrics and Gynecology (ISUOG) (4).

$\mathrm{La}$ incidencia de ventriculomegalia aislada es de 0,64/1000 embarazos (5-7). El pronóstico de la ventriculomegalia depende de la existencia de otras malformaciones o síndromes asociados, en caso de hallazgo aislado, es más desfavorable en fetos de sexo femenino. En este estudio se han recopilado retrospectivamente casos de ventriculomegalia diagnosticados en nuestro centro en los últimos cinco años, para establecer la relación entre ventriculomegalia y pronóstico perinatal.

\section{PACIENTES Y MÉTODO}

Se estudiaron retrospectivamente 60 casos diagnosticados de ventriculomegalia en nuestra Unidad de Ecografía entre enero de 2006 y enero de 2011. Se tomaron como criterios ecográficos de ventriculomegalia la medida del asta posterior de los ventrículos laterales mayor de $10 \mathrm{~mm}$, siguiendo criterio de la ISUOG (4).

Una vez diagnosticada, y con el consentimiento de la paciente, se solicitó estudio del cariotipo, serológico (en líquido amniótico y sangre materna) de toxoplasma, rubeola, citomegalovirus, parvovirus B19, y herpes virus. Además se practicó una ecografía morfológica exhaustiva para descartar anormalidades asociadas. Finalmente, fue solicitada una valoración conjunta con el pediatra neurólogo. A aquellas pacientes con criterios de mal pronóstico (ventriculomegalia severa precoz, progresiva, malformaciones asociadas, aneuploidías, o anomalías en las pruebas referidas) se les infor- mó sobre la posibilidad de finalizar la gestación. En el resto de los casos se estableció un seguimiento inicial semanal, para luego evaluar cada 2-3 semanas los procesos que se mantuvieron estables. En algunos casos, se completó el estudio de imagen con resonancia magnética. El seguimiento de las gestaciones se realizó en la consulta de obstetricia de alto riesgo. La vía del parto se decidió por motivos estrictamente obstétricos.

\section{RESULTADOS}

La edad gestacional media al diagnóstico fue de 24 semanas (rango: 17-37 semanas). La edad media de la madre en el momento del diagnóstico era de 29 años (rango: 15-41 años). En 2 pacientes la gestación fue conseguida mediante técnicas de reproducción asistida y 3 pacientes presentaban antecedentes de malformaciones en gestaciones anteriores (1 recién nacido con estenosis pulmonar y 2 con aborto legal por síndromes polimalformativos).

En 6 pacientes se asoció una aneuploidía (10\%) (4 trisomías 21, 1 triploidía y 1 trisomía 13). En 2 casos hubo una infección materna (citomegalovirus y lúes). Además, 16 pacientes (27\%) presentaban otras anomalías asociadas (Figura 1).

Decidieron interrumpir la gestación 23 pacientes $(38 \%)$. Si se consideran sólo las gestaciones cuyo diagnóstico se consiguió con edad gestacional dentro del marco legal ( $\leq 22$ semanas), se acogieron a la ley vigente el $74 \%$. De las que interrumpieron el embarazo presentaban: 6 aneuploidías, 1 lúes positiva, malformaciones asociadas (Figuras 2 y 3) y el resto desarrollaron una ventriculomegalia progresiva.

En aquellas que continuaron con el embarazo, la ventriculomegalia fue progresiva en 6 casos. De estas últimas, hubo 2 casos de muerte intraútero, el otro tiene actualmente 18 meses sin presentar alteraciones del desarrollo cognitivo (Figura 3), el cuarto fue exitus a los 7 meses de vida por un síndrome de Miller Dieker de diagnóstico postnatal, el quinto fue exitus a los tres meses de vida por un síndrome Marden Walker de diagnóstico postnatal (síndrome polimalformativo congénito más una enfermedad por reflujo gastroesofágico, incluye afectación de la vía aérea superior y enfermedad por reflujo gastroesofágico grave) y el sexto presenta secuelas severas secundarias a la infección por citomegalovirus durante el tercer trimestre (hipoacusia, retraso mental, etc.). En el síndrome de Miller Dieker no se detectó prenatalmente la lisencefalia y el cuadro fue etiquetado de ventriculomegalia leve con polihidramnios. 


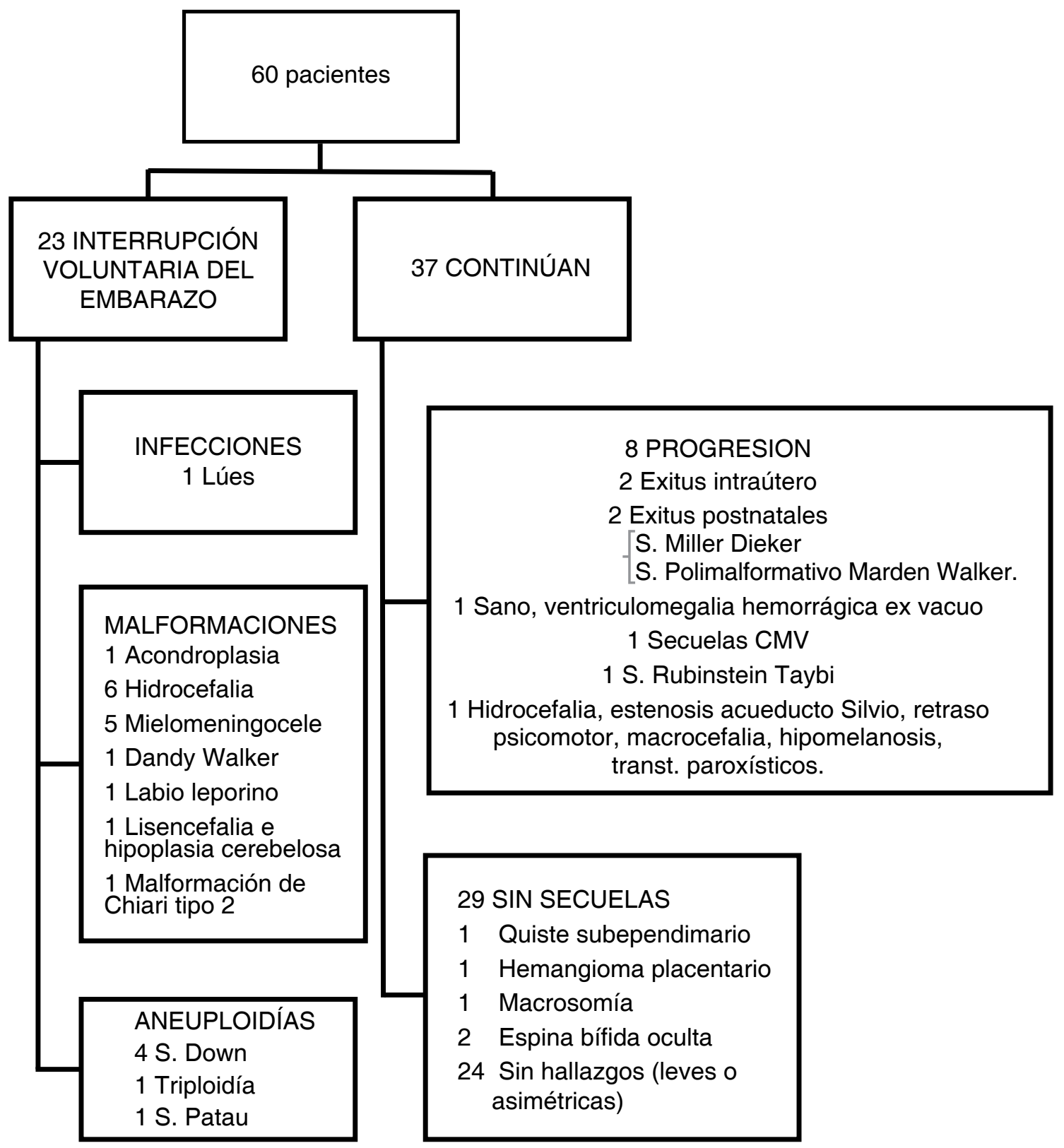

Figura 1. Esquema que representa el seguimiento y destino de la gestación de las pacientes con medida de astas posteriores de ventrículos laterales cerebrales fetales mayor de $10 \mathrm{~mm}$.

De los casos que continuaron con la gestación y que presentaban una ventriculomegalia leve y estable, se diagnosticó posnatalmente 2 casos de espina bífida oculta que fueron tratadas quirúrgicamente y 1 recién nacido con un quiste subependimario sin complicaciones asociadas.

En todos los casos el momento de la finalización del parto se produjo al término y la vía del parto fue vaginal en el $82 \%$ (17 partos normales, 6 fórceps y 11 cesáreas). 


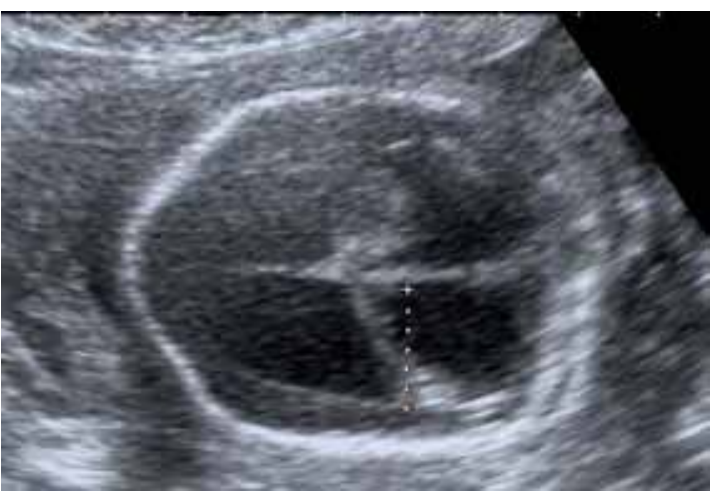

Figura 2. Ventriculomegalia grave en una gestación de 20 semanas coexistiendo con un mielomeningocele.

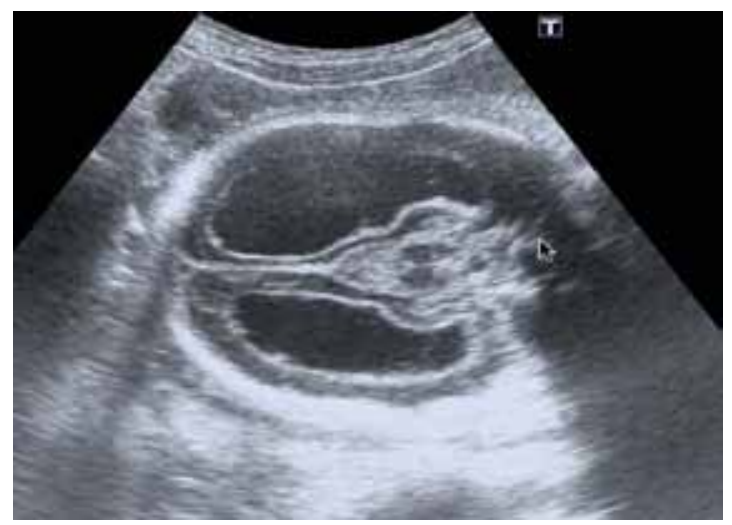

Figura 3. Ventriculomegalia grave en una gestación de 20 semanas en la que postnatalmente la necropsia diagnosticó una lisencefalia.

\section{DISCUSIÓN}

Del total de 60 ventriculomegalias, 28 corresponden a recién nacidos sin secuelas (47\%). Nuestra casuística, aunque no comparable en número con la de Larskin y cols (14), aporta resultados inferiores respecto al pronóstico favorable, para estos autores el $79 \%$ de los casos de ventriculomegalias aisladas corresponden a desarrollos neonatales normales. Probablemente la diferencia esté en la cifra de casos estudiados. Por otra parte, excluyendo los casos con malformaciones asociadas (16 casos), nuestra cifra de resultados favorables es del $70 \%$, poniéndose así de manifiesto que las diferencias en la literatura son debidas parcialmente a los criterios de inclusión y exclusión de los casos.

Para evitar los falsos negativos y positivos, y la variabilidad interobservador en la ecografía de la semana 20 se deben seguir protocolos estrictos de medición. Es importante medir el ventrículo lateral en la dirección del eje y con los caliper de dentro a dentro para evitar crear un error de hasta el $10 \%$. La cantidad de plexo coroideo hiperecoico en los ventrículos puede ayudar en casos límite. En el atrio, los plexos coroideos normalmente llenan $>60 \%$. En una verdadera ventriculomegalia los plexos coroideos disminuyen en tamaño (relativo al atrio) y pueden también "colgar" hacia abajo Es preciso tener en cuenta que en fetos varones estas estructuras son ligeramente más grandes que en las mujeres, para cada edad gestacional.

En la mayoría de los casos registrados en la literatura la presencia de una ventriculomegalia moderada aislada no tiene repercusión clínica importante $(1,5,8-10)$. Rosseau y cols (9), concluyen que sólo en un $4,5 \%$ de los casos la ventriculomegalia es progresiva, cifra que en nuestro caso fue casi el doble $(10 \%)$. Por tanto, es importante la realización de controles ecográficos para determinar si el proceso es estable o progresivo, si se asocia a nuevos hallazgos, o se resuelve espontáneamente. Por otro lado, la incidencia de malformaciones asociadas presenta cifras de hasta el $84 \%(9,11,12)$, que en nuestra serie alcanzó el $45 \%$, excluyendo las aneuploidías. La importancia de esta cifra la ilustran Pober y cols (13), quienes determinan que el principal factor que influye en el pronóstico es la presencia de otras malformaciones asociadas.

En esta serie se observó que la medida de la ventriculomegalia no permite predecir el resultado perinatal. El diagnóstico prenatal de ventriculomegalia obliga al clínico a la búsqueda exhaustiva de otras anomalías. Por esta razón, aunque controvertida, se recomienda el uso complementario de la RMN en aquellos casos donde la ecografía es técnicamente difícil (oligoamnios, obesidad materna, etc.).

De igual forma debe valorarse la realización del estudio citogenético fetal. La cromosomopatía más frecuentemente asociada a esta condición es el síndrome de Down. En nuestro trabajo hemos encontrado un $10 \%$ de cariotipos anormales, cifras similares a las encontradas en la literatura $(11,15)$. Terry y cols (16), consideran que la venticulomegalia aislada es un marcador ecográfico asociado a aneuploidías.

Finalmente, deberemos incluir el estudio microbiológico materno-fetal para descartar infecciones tales como el citomegalovirus, sífilis o toxoplasmosis. En un pequeño número de pacientes, la presencia de una ventriculomegalia puede ser la primera manifestación de un daño cerebral consecuencia de una infección (12). 
El recién nacido diagnosticado de una ventriculomegalia requiere de un detallado examen físico. En ocasiones deberá acompañarse de otras pruebas de imagen como la RMN, la ecografía transfontanelar o la TAC. Lee y cols (17), encontraron que en más del $50 \%$ de los fetos tenían asociada otras anomalías en el sistema nervioso central (SNC), de éstas sólo el $12 \%$ fueron diagnosticadas prenatalmente. Ellos hacen hincapié en la búsqueda de otras malformaciones neurológicas en la vida postnatal. En nuestro trabajo no se diagnosticaron prenatalmente 1 lisencefalia, 2 espinas bífidas ocultas, 1 quiste subependimario y 1 estenosis del acueducto de Silvio. La RMN se solicitó en 11 casos en los que la resolución ecográfica no fue óptima (una sospecha de Dandy Walker que se confirmó, tres asimetrías ventriculares, una imagen sugestiva de mielomeningocele que correspondió a una malformación de Chiari tipo 2, dos informadas como normales, una de las cuales se correspondió con hidrocefalia secundaria a estenosis del acueducto de Silvio [asociando macrocefalia, hipomelanosis, retraso psicomotor...] y cuatro ventriculomegalias moderadas sin otros hallazgos) y el resultado no determinó actuación diferente a la ya pautada.

En un estudio realizado por Váradi y cols (18), encontraron un $4 \%$ de recurrencia del total de casos. Recientemente, con el desarrollo de la genética molecular, se ha encontrado una serie de enfermedades con herencia recesiva ligada al cromosoma $X$, que incluye a la hidrocefalia como una de las manifestaciones. Se cree que la hidrocefalia relacionada al cromosoma $X$ representa casi un $5 \%$ de la totalidad de casos $(19,20)$, aunque debido probablemente al tamaño de la muestra no encontramos ninguno.

El resultado postnatal depende fundamentalmente de la edad gestacional al diagnóstico, el diámetro de las astas posterior y su progresión, el sexo, el cariotipo, la serología y la presencia de otras malformaciones asociadas. En esta serie el $47 \%$ de las pacientes con un diagnóstico de ventriculomegalia tuvieron un recién nacido sin secuelas neurológicas, cifra ésta que sube al $70 \%$ si se excluyen los casos con malformaciones asociadas. Por tanto, se encuentra acuerdo con la opinión de muchos autores (17) que si la ventriculomegalia no es progresiva, ni se asocia a otras malformaciones, la probabilidad de tener un recién nacido sano es superior al $70 \%$. La gran variabilidad entre publicaciones hace preciso que cada centro disponga de sus propios datos en función de sus medios, para ofrecer consejo a los padres que han de decidir el futuro de la gestación.

\section{REFERENCIAS}

1. Ouahba J, Luton D, Vuillard E, Garel C, Gressens P, Blanc N, Elmaleh M, Evrard P, Oury JF. Prenatal isolated mild ventriculomegaly: outcome in 167 cases. BJOG 2006;113:1072-9.

2. Wyldes $M$, Watkinson $M$. Isolated mild fetal ventriculomegaly. Arch Dis Child Fetal Neonatal Ed 2004;89:F913.

3. Holzgreve W, Feil R, Louwen F, Miny P. Prenatal diagnosis and management of fetal hydrocephaly and lissencephaly. Childs Nerv Syst 1993;9:408-12.

4. Sonographic examination of the fetal central nervous system: guidelines for performing the 'basic examination' and the 'fetal neurosonogram'. Ultrasound Obstet Gynecol 2007;29:109-16.

5. Joo JG, Toth Z, Beke A, Papp C, Toth-Pal E, Csaba A, Szigeti Z, Rab A, Papp Z. Etiology, prenatal diagnostics and outcome of ventriculomegaly in 230 cases. Fetal Diagn Ther 2008;24:254-63.

6. Wilson RD, Hitchman D, Wittman BK. Clinical followup of prenatally diagnosed isolated ventriculomegaly, microcephaly and encephalocele. Fetal Ther 1989;4:49-57.

7. Wiswell TE, Tuttle DJ, Northam RS, Simonds GR. Major congenital neurologic malformations. A 17-year survey. Am J Dis Child 1990;144:61-7.

8. Valat AS, Dehouck MB, Dufour P, Dubos JP, Djebara AE, Dewismes L, Robert Y, Puech F. [Fetal cerebral ventriculomegaly. Etiology and outcome, report of 141 cases]. J Gynecol Obstet Biol Reprod (Paris) 1998;27:782-9.

9. Rosseau GL, McCullough DC, Joseph AL. Current prognosis in fetal ventriculomegaly. $\mathrm{J}$ Neurosurg 1992;77:551-5.

10. Monteagudo A, Timor-Tritsch IE, Moomjy M. In utero detection of ventriculomegaly during the second and third trimesters by transvaginal sonography. Ultrasound Obstet Gynecol 1994;4:193-8.

11. Bromley B, Frigoletto FD, Jr., Benacerraf BR. Mild fetal lateral cerebral ventriculomegaly: clinical course and outcome. Am J Obstet Gynecol 1991;164:863-7.

12. Graham E, Duhl A, Ural S, Allen M, Blakemore K, Witter $F$. The degree of antenatal ventriculomegaly is related to pediatric neurological morbidity. J Matern Fetal Med 2001;10:258-63.

13. Pober BR, Greene MF, Holmes LB. Complexities of intraventricular abnormalities. J Pediatr 1986;108:54551.

14. Laskin MD, Kingdom J, Toi A, Chitayat D, Ohlsson A. Perinatal and neurodevelopmental outcome with isolated fetal ventriculomegaly: a systematic review. J Matern Fetal Neonatal Med 2005;18:289-98.

15. Achiron R, Schimmel M, Achiron A, Mashiach S. Fetal mild idiopathic lateral ventriculomegaly: is there a correlation with fetal trisomy? Ultrasound Obstet Gynecol 1993;3:89-92.

16. Terry M, Calhoun BC, Walker W, Apodaca C, Martin L, Pierce B, Hume RF, Evans MI. Aneuploidy and isolated mild ventriculomegaly. Attributable risk for isolated fetal marker. Fetal Diagn Ther 2000;15:331-4. 
17. Lee CS, Hong SH, Wang KC, Kim SK, Park JS, Jun JK, Yoon BH, Lee YH, Shin SM, Lee YK, Cho BK. Fetal ventriculomegaly: prognosis in cases in which prenatal neurosurgical consultation was sought. J Neurosurg 2006;105:265-70.

18. Papp C, Adam Z, Toth-Pal E, Torok O, Varadi V, Papp Z. Risk of recurrence of craniospinal anomalies. J Ma- tern Fetal Med 1997;6:53-7.

19. Schrander-Stumpel CT, Howeler CJ, Fryns JP. Xlinked mental retardation and neurological symptoms: a nosological approach. Genet Couns 1995;6:21-32.

20. Schrander-Stumpel CT, Vos YJ. [From gene to disease; X-linked hydrocephalus and LiCAM]. Ned Tijdschr Geneeskd 2004;148:1441-3. 